\title{
A field cage test of the effects of the endosymbiont Wolbachia on Drosophila melanogaster
}

\author{
KAREN OLSEN, K. TRACY REYNOLDS \& ARY A. HOFFMANN* \\ Centre for Environmental Stress and Adaptation Research, La Trobe University, Bundoora, Victoria 3083, Australia
}

\begin{abstract}
Wolbachia endosymbionts are known to affect the fitness of their hosts, but most of this information is from laboratory studies. In Drosophila melanogaster, Wolbachia frequencies vary clinally in frequency in Australia and may confound climatic adaptation. Here we use field cages in a reciprocal exchange design to test for Wolbachia effects in D. melanogaster in winter at temperate and tropical sites. Infected flies of both populations had a lower fecundity in tropical north Queensland, whilst in temperate southern Victoria Wolbachia effects depended on the nuclear population background. Here infected flies from Victoria were more fecund. Wolbachia also influenced larval/pupal viability in the tropics but this was dependent on population background. In comparisons of the populations, there was no evidence for local adaptation for total fecundity, viability or survival over winter. However, in Victoria, a local population had a higher late-life fecundity than a tropical population from Queensland that had higher early-life fecundity. At a tropical site, local Queensland flies had a higher early fecundity than Victorian flies. In contrast to many laboratory studies, mortality rates in the field cages increased only slightly over time. Both the Wolbachia effects and population differences have not been previously detected in laboratory studies with $D$. melanogaster and highlight the utility of Drosophila field studies in fitness experiments.
\end{abstract}

Keywords: Drosophila, field fitness, maternal transmission, symbiont, Wolbachia.

\section{Introduction}

Wolbachia are common endosymbionts of insects (Werren, 1997; Jeyaprakash \& Hoy, 2000) that are responsible for cytoplasmic incompatibility in Drosophila (Hoffmann \& Turelli, 1997), which involves embryo death when infected males mate with uninfected females or when matings take place between strains with different infections. A number of laboratory experiments have established that Wolbachia can have other impacts on the fitness of their hosts. For instance, Wolbachia can cause deleterious effects on Drosophila (Hoffmann et al., 1990), Trichogramma (Schoenmaker et al., 1998) and mites (Johanowicz \& Hoy, 1999). By contrast, there is almost no information on fitness effects of Wolbachia under field conditions. Field studies on transmission dynamics and the cytoplasmic incompatibility generated by Wolbachia indicate that parameter estimates for these variables can differ markedly from those estimated under laboratory conditions (Turelli \&

\footnotetext{
*Correspondence. E-mail: A.Hoffmann@latrobe.edu.au
}

Hoffmann, 1995). Unfortunately it is difficult to measure fitness effects of Wolbachia under field conditions and only indirect inferences have been possible (Hoffmann et al., 1998).

Here we examine fitness effects of Wolbachia in Drosophila melanogaster. In this species, one Wolbachia has been identified which causes a low level of incompatibility in laboratory crosses and occurs at different frequencies in populations (Hoffmann et al., 1994; Hoffmann et al., 1998). This is especially marked in eastern Australia, where northern populations are mostly infected and southern populations are mostly uninfected (Hoffmann et al., 1994). However, the persistence of Wolbachia in populations is a mystery because D. melanogaster Wolbachia are transmitted imperfectly across generations and in the absence of strong incompatibility in the field, the infection is expected to decrease in frequency (Hoffmann et al., 1998). One possible explanation is that Wolbachia have positive fitness effects on their hosts, and the finding that Wolbachia can alter host fly size in some field conditions (Hoffmann et al., 1998) suggests host fitness effects. 
To investigate Wolbachia effects, populations were set up with flies collected from tropical (north Queensland) and temperate (Victoria) locations in eastern Australia. By testing the infection status of isofemale lines and by undertaking reciprocal crosses, infected and uninfected populations from both locations were established. Populations were then set up in field cages and tested for fitness components over winter in Victoria and Queensland. This design allowed a direct assessment of Wolbachia effects on host fitness and interactions between the nuclear background and Wolbachia as well as overall differences between the background populations. Population cages in Victoria were maintained until reproduction ceased so that lifetime reproduction and mortality patterns over winter could be investigated. The maternal transmission efficiency of Wolbachia in Victoria was also examined by scoring the infection status of offspring of field-reared females.

\section{Materials and methods}

\section{Flies and Wolbachia status}

Flies were collected in early March 1999 from fruit orchards in Wandin, Victoria (40 km east of Melbourne). Adults were netted from rotting fruit piles containing nectarines, peaches and apples. Inseminated females were used to set up isofemale lines. To establish the presence or absence of Wolbachia within these lines, PCR analysis was performed on the field females using 16S rDNA primers (O’Neill et al., 1992) following procedures for population monitoring of Wolbachia infections in D. melanogaster (Hoffmann et al., 1998). To ensure that Wolbachia negative results were not due to a lack of DNA template or PCR failure, primers for the single copy Drosophila nuclear gene suppressor of sable su(s) (Voelker et al., 1991) were also included in each reaction. Progeny from each line were also tested to verify infection status. From 107 lines screened, eight infected lines were found. These were combined to establish an infected population and nine uninfected lines were also combined to form an uninfected population. The infected and uninfected populations were then reciprocally crossed to remove any genetic background effects. These populations are henceforth referred to as infected and uninfected Melbourne populations. The same procedure (with the same number of infected and uninfected lines) was also used to establish infected and uninfected flies from Innisfail, Queensland (92 km south of Cairns, henceforth referred to as Innisfail populations). $F_{1} s$ from the reciprocal crosses were collected to initiate the field population cages.

\section{Victoria experiment}

Field cages were constructed from $1.5 \mathrm{~L}$ plastic containers. Two sides were removed and covered with gauze, and food was provided from sucrose-agar-dead yeast medium in plastic containers, $7 \mathrm{~cm}$ in diameter and $4.5 \mathrm{~cm}$ high. Each cage was set up with 15 females and 15 males from one of the four populations. There were 12 replicate cages for each population. The cages were placed under plastic ventilated baskets (five cages per basket) for shade and protection, to create 10 blocks. The position of the plastic baskets was rotated every 3-5 days. Cages were placed in shade under trees at the Bundoora campus of La Trobe University on 2 June 1999. Temperatures were continuously recorded using a data logger (Tinytalk II) placed inside one of the cages. Previous recordings had shown that temperatures inside and outside the cages were almost identical. The experiment was terminated in early November when almost all flies were dead and there was no further oviposition.

To score survival, dead flies were removed from cages every $2-5$ days and frozen. To assess fecundity, food cups were replaced in the cages every $2-5$ days and the number of eggs laid in each cup was recorded. Egg hatch rates and larval/pupal viability were assessed on 7 June, when 10 eggs were removed from each food cup and placed in a separate vial containing $10 \mathrm{~mL}$ of the laboratory medium. Each vial was then covered by fine gauze. The vials were placed into cages, and these cages were then placed under plastic baskets as for the population cages. The number of eggs that hatched and number of eclosed adults was used to assess egg hatch rate and viability, respectively.

Maternal transmission of Wolbachia was measured on six females reared from eggs placed in the field. One egg laid on 19 June and 5 eggs laid between 12 and 22 October produced infected adult females. The transmission efficiency of Wolbachia in each female (as measured by the proportion of uninfected progeny) was estimated by rearing 30 offspring per female under laboratory conditions and determining their Wolbachia status by PCR.

\section{Queensland experiment}

The site used for this experiment was a remnant area of rainforest near Innisfail, Queensland near the original collection site of the Innisfail flies. Cages were established in shade under trees at the same time as the cages in Victoria. However, cages were only left for two weeks and food cups were replaced daily to compensate for much higher egg counts. Eggs were counted to obtain a fecundity score and survival was also monitored, 
although only a few flies ( $<10 \%$ per cage) died and survival data are not considered further. Egg hatch rate and larval/pupal viability were also scored as described above.

\section{Results}

\section{Victoria experiment}

Egg hatch rates did not differ between treatments (Table 1) and were low (Fig. 1) reflecting the fact that environmental conditions were stressful for eggs. None of the hatched larvae developed to the adult stage. We also monitored larvae hatching in the cups in July, August and October. Only in the case of the October larvae did a substantial proportion (16/25 or $64 \%)$ develop to the adult stage. Presumably higher temperatures are required for eggs to develop successfully to the adult stage. For June, the mean temperature in the cages was $10.6^{\circ} \mathrm{C}$ (minimum $0.2^{\circ} \mathrm{C}$ ), compared to $11.1^{\circ} \mathrm{C}$ $\left(1.1^{\circ} \mathrm{C}\right), 13.1^{\circ} \mathrm{C}\left(1.1^{\circ} \mathrm{C}\right), 14.1^{\circ} \mathrm{C}\left(4.6^{\circ} \mathrm{C}\right)$, and $14.5^{\circ} \mathrm{C}$ $\left(3.5^{\circ} \mathrm{C}\right)$ for August, September, October and early November, respectively. Unfortunately no data were collected for July.

Mean survival times of the flies were computed for each cage and compared among the treatments. Neither infection status nor population origin had any impact on this trait (Table 1). To examine mortality patterns further, we computed the cumulative mortality separately for each cage based on data from the different time intervals. Cumulative mortality showed a similar pattern in all treatments (Fig. 2) and was near 100\% at the end of the experiment. The mortality pattern was further examined by computing mortality rates at time $x\left(\mu_{x}\right)$ following Promislow et al. (1996) defined as $-\ln \left(N_{x+1} /\right.$ $N_{x}$ ), where $N$ is the number of flies alive at a particular time. The mortality rate was adjusted to account for the different number of days in the scoring interval. Mean mortality rates for the cages did not change much in all the treatments (Fig. 2) although there was a gradual increase in mortality rate as the experiment progressed. Linear regression showed that this link was significant $(P<0.01)$ for both the infected $(y=0.0075 x-4.86)$ and uninfected $(y=0.0063 x-4.79)$ Innisfail populations as
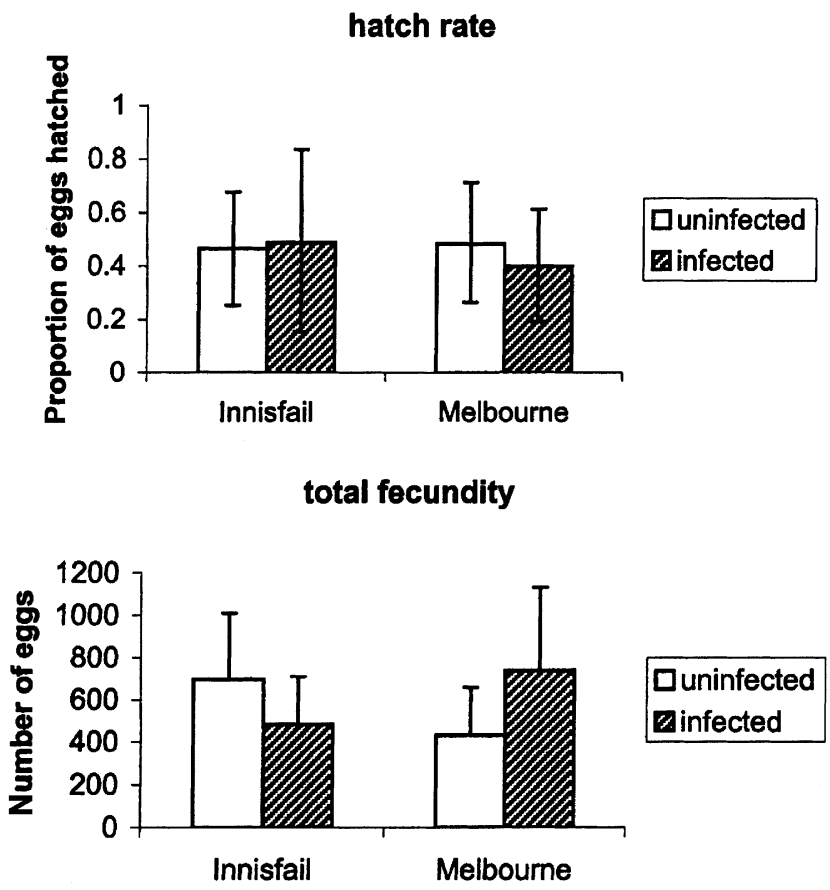

Fig. 1 Means and standard deviations (error bars) for hatch rate and total fecundity in Drosophila melanogaster for the experiments in Victoria. Hatch rates were measured on groups of 10 eggs set up in June and there were 6-10 replicate vials per treatment. Standard deviations for hatch rates are based on arcsin transformations (re-expressed as proportions). Total fecundity means are based on 12 cages per treatment.

Table 1 Analyses of variance testing effects of infection status and geographical origin on fitness measures undertaken on Drosophila melanogaster from cages placed in Victoria and Queensland. Table entries are mean squares

\begin{tabular}{lcccr}
\hline Trait & Infection (d.f. =1) & Origin (d.f. =1) & Interaction (d.f. =1) & Error (d.f.) \\
\hline Victoria & & & & \\
$\quad$ Survival time & 0.07 & 86.91 & 221.24 & $252.91(44)$ \\
$\quad$ Total fecundity $\times 10^{-4}$ & 26.88 & 0.19 & $816.41^{* *}$ & $88.16(44)$ \\
$\quad$ Time to half egg lay & 844.93 & $1880.75^{* *}$ & 39.10 & $219.16(44)$ \\
Hatchability (arcsine) $\times 10^{2}$ & 3.39 & 0.01 & 9.41 & $6.99(27)$ \\
Queensland & & & & \\
$\quad$ Total fecundity $\times 10^{-4}$ & $26.81^{* *}$ & $11.35^{*}$ & $9.46^{*}$ & $2.30(43)$ \\
Hatchability (arcsine) $\times 10^{2}$ & 14.22 & 0.19 & 1.06 & $4.09(39)$ \\
Viability (arcsine) $\times 10^{2}$ & 27.73 & 16.05 & $86.71^{* *}$ & $6.92(39)$ \\
\hline
\end{tabular}

$* P<0.05 ; * * P<0.01$.

(c) The Genetics Society of Great Britain, Heredity, 86, 731-737. 
fecundity

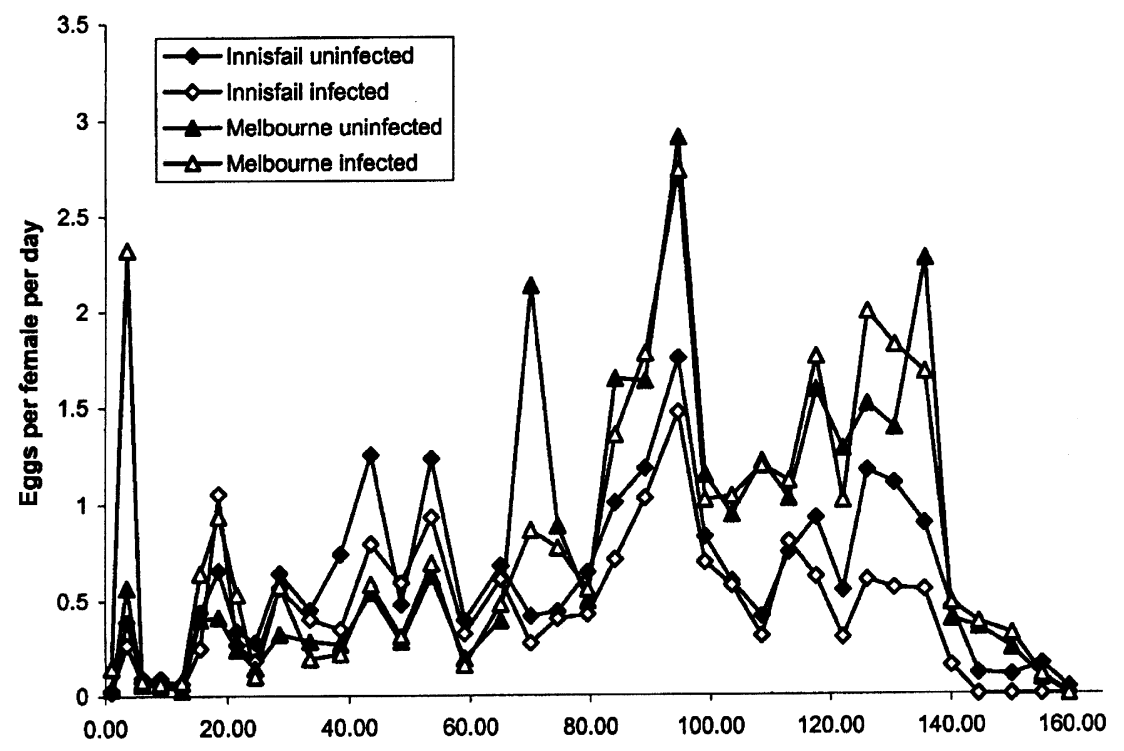

cumulative mortality

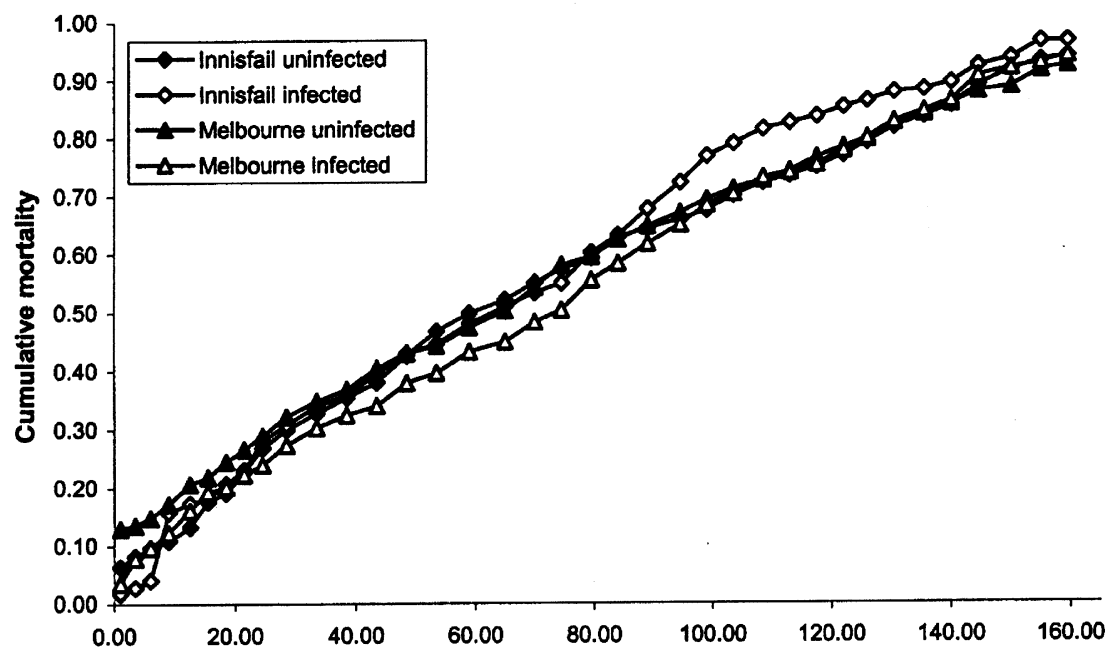

Fig. 2 Fecundity and survival patterns of Drosophila melanogaster in cages set up in Victoria. Fecundity is expressed as the number of eggs produced per female, taking into account the number of days over which fecundity was scored. Survival data are presented as cumulative mortality in the cages and as mortality rate computed as described in the text. Each data point represents the mean of 12 cages. well as the infected $(y=0.0069 x-4.86)$ and uninfected $(y=0.00062 x-4.87)$ Melbourne populations.

The total fecundity in the cages depended on both infection status and population origin, as the interaction between these factors was significant in the ANOVA (Table 1). For the Innisfail flies the uninfecteds had a relatively higher overall fecundity whereas the opposite result was obtained for the Melbourne flies. In both population backgrounds there were significant differences between the infecteds and uninfecteds.

Fecundity patterns over time were also examined by computing egg counts separately for each cage and time interval and then adjusting for the number of females alive in a cage within that interval. The per female oviposition rates showed parallel changes in all treatments (Fig. 2) and increases matched brief periods of warmer weather. For instance the peak at 94.5 days coincided with a period when the mean temperature was $22.1^{\circ} \mathrm{C}$ compared to $16.2^{\circ} \mathrm{C}$ in the ensuing period when fecundity decreased sharply. There was also a gradual increase in fecundity in the first 90 days or so which was followed by a decline until almost no eggs were produced by the flies surviving to 160 days. The graph also indicates a difference in the fecundity pattern of the Melbourne and Innisfail flies. Towards the end of the experiment, Melbourne flies produced more eggs than Innisfail flies, whereas the opposite trend is apparent at the start of the experiment (Fig. 2). To test this 
statistically, we computed the time taken for females in each cage to lay half their eggs. The ANOvA on these values indicated a significant effect of population background but not infection on this trait (Table 1) reflecting the later oviposition pattern of the Melbourne flies.

For the transmission rate experiment, a total of 19 uninfected individuals were identified from 166 progeny. Transmission rates varied among the females, ranging from $70.4 \%$ to $100 \%$. The lowest transmission rate was observed for the single infected female that emerged from eggs laid in June, which took 86 days to eclose. Differences among females were significant by a contingency test $(P=0.010$ using the Monte Carlo procedure). This procedure was used because of the low number of expecteds in many cells. Confidence intervals based on the population transmission rate of $89 \% \quad(83-93 \%)$ do not overlap previous estimates of maternal transmission of $97.4 \% \quad(95 \%$ CIs of 94.1-99.2\%) (Hoffmann et al., 1998). Thus maternal transmission rates are lower than previously observed in D. melanogaster.

\section{Queensland experiment}

For egg hatch rates, there were no differences among treatments (Table 1) and hatch rates were high (Fig. 3) compared to those observed in Victoria. This is likely to reflect the higher temperatures experienced at the Queensland site. Over the two-week period, temperatures ranged from $17.3^{\circ} \mathrm{C}$ to $25.3^{\circ} \mathrm{C}$ with a mean of $21.1^{\circ} \mathrm{C}$. For larval-to-adult viability, the interaction between infection status and origin was significant. For Innisfail flies, uninfected larvae showed the highest viability, whereas the reverse pattern was evident for the Melbourne flies, although for the Melbourne background the difference between infecteds and uninfecteds was not significant.

Fecundity data were collected over two weeks only in this experiment. Because differences among treatments were consistent within this period only the results of pooled egg counts are considered. The ANOvA indicated a significant interaction effect as well as significant main effects of population origin and infection (Table 1). Uninfected flies had a higher fecundity than infected flies and this difference was relatively larger in the Innisfail background (Fig. 3). Overall, egg production was higher for the populations originating from Queensland compared to those from Victoria.

\section{Discussion}

The comparisons of infected and uninfected populations suggest that Wolbachia effects on fitness depend on

\section{hatch rate}
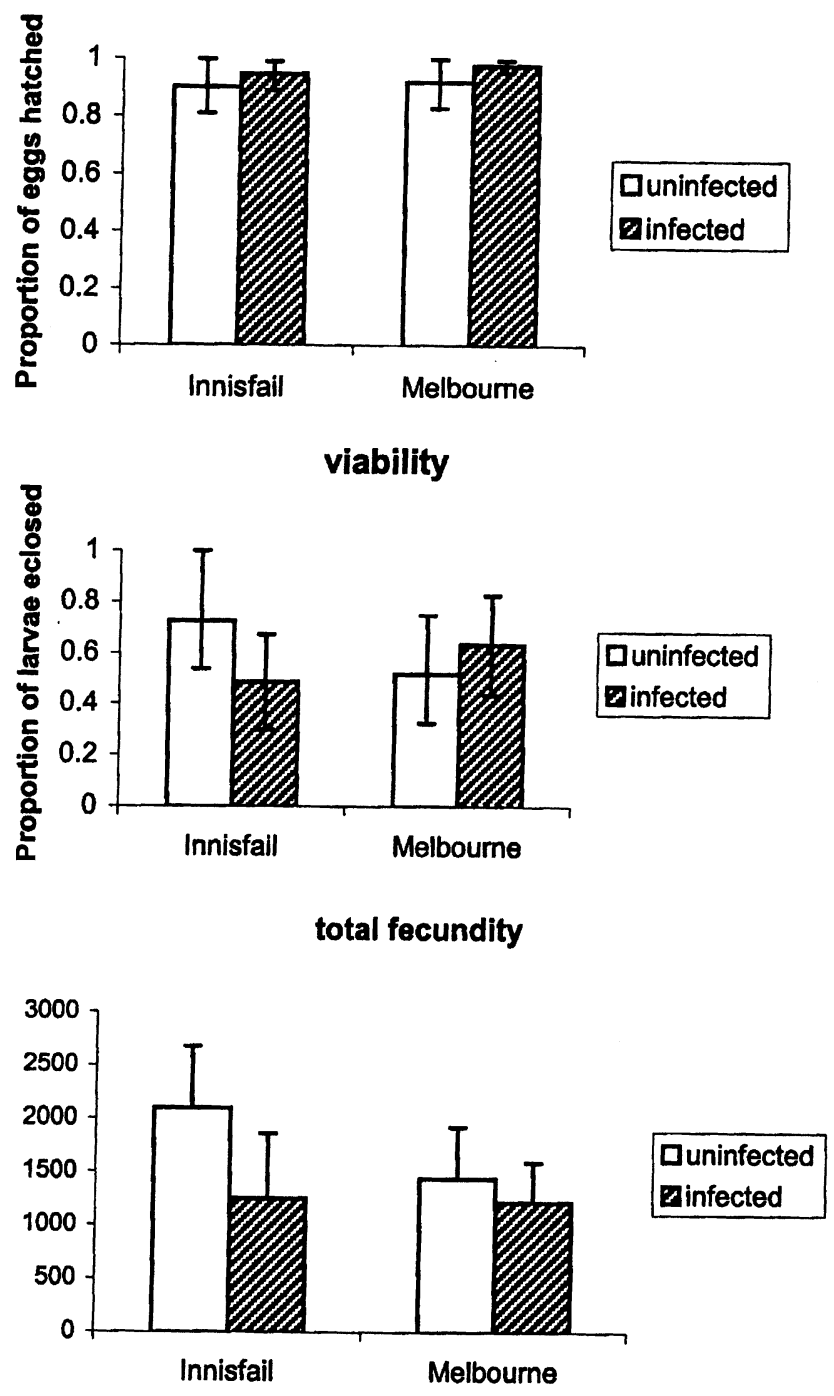

Fig. 3 Hatch rate, larval/pupal viability, and total fecundity in Drosophila melanogaster for the experiments in Queensland. Means and standard deviations (error bars) are based on 10 (hatch rate and viability) or 12 replicates. Standard deviations for hatch rates and viability are based on arcsin transformations (re-expressed as proportions).

nuclear background. In previous laboratory studies on Drosophila, which have generally been carried out on only one host background, Wolbachia has been shown to decrease fecundity (Hoffmann et al., 1990) or have little impact on fecundity (Hoffmann et al., 1994; Giordano et al., 1995; Bourtzis et al., 1996; Mercot, 1997). The present results suggest that Wolbachia may also increase fecundity given the appropriate environmental conditions (temperate winter) and nuclear background (Melbourne). In a previous laboratory experiment on the $D$. melanogaster infection there was 
no impact of Wolbachia on fecundity in the laboratory (Hoffmann et al., 1994). Because the infected Innisfail females in the present experiments showed a reduction in fecundity in contrast to the laboratory findings, this raises the issue of whether the laboratory experiments are particularly informative in understanding the field situation.

The Wolbachia effects on host fitness do not account for the clinal variation present along the east coast of Australia. Because there is a low incidence of Wolbachia in Melbourne and other southern populations (Hoffmann et al., 1994; Hoffmann et al., 1998), we anticipated a reduced fitness of infected individuals at this location particularly in the Melbourne nuclear background. Instead, we observed a reduction in total fecundity for infected individuals but only in the Innisfail nuclear background. We also anticipated a fitness advantage for Wolbachia-infected flies in Queensland, but instead obtained the opposite result.

We can only surmise that these host effects must be offset by other factors to account for the distribution of Wolbachia. The relatively lower rate of maternal transmission of the infection in females reared in winter may contribute to the low infection frequency in Melbourne. Previous work on Nasonia wasps has also shown that overwintering reduces the efficiency of maternal transmission (Perrot-Minnot et al., 1996). However, the transmission rate of Wolbachia in field D. melanogaster is also imperfect in warmer locations, so the infection should still be lost from all populations. Perhaps the effect of Wolbachia on fecundity are offset by effects on other traits, such as starvation or desiccation resistance which are negatively genetically correlated with early reproductive output (Hoffmann \& Harshman, 1999).

The data also suggest geographical differences between $D$. melanogaster populations from the tropics and temperate areas for fecundity patterns, although these findings should be regarded as preliminary without information from other populations. By postponing egg laying, Melbourne females may increase the likelihood of laying eggs in warmer spring temperatures. This could be adaptive given that overwintering D. melanogaster in temperate areas tend to be adults (Izquieredo, 1991), as evident here from the high mortality of eggs and absence of successful larval/pupal development to the adult stage. Bouletreau-Merle et al. (1992) have argued that population variation in egg retention capacity and a seasonal increase in spring in retention capacity reflects selection for overwintering. The different fecundity patterns observed here may be related to retention capacity although a genetic correlation between virgin retention and late-life reproduction has not been established. It is not clear if these differences in fecundity patterns depend on climatic conditions. In Queensland, the tropical population had a higher fecundity and this might have resulted in a relatively lower fecundity later in life. It is possible that early fecundity is favoured in tropical conditions, but this remains to be demonstrated.

The survival data provide no evidence for a trade-off between early reproduction and survival over winter in D. melanogaster because shifts in fecundity patterns did not influence survival. A trade-off between these traits is evident from laboratory selection experiments on postponed senescence (e.g. Rose \& Charlesworth, 1981; Partridge et al., 1999). Perhaps trade-offs are only detected when reproductive output is high as in favourable laboratory conditions. In winter in Victoria, the egg production we observed was extremely low; the total number of eggs over the entire 165-day period was much lower than that produced over only two weeks in Queensland (see Figs 1, 3). Thus in winter field conditions where survival is favoured there may be no trade-off with early fecundity because low temperatures restrict reproductive output. Nevertheless, differences between the Melbourne and Innisfail cages are consistent with a trade-off between early and late reproductive output, since Melbourne flies had a relatively lower early fecundity and higher later fecundity.

The pattern of mortality in the field does not match that seen in laboratory experiments on D. melanogaster. In these experiments there is generally an increase in mortality rates over time, which levels off at advanced ages (Fukui et al., 1993; Promislow et al., 1996). This contrasts with the mortality pattern in the field cages, where there was only a small increase in mortality rate over time. The way mortality rates change with age are likely to be environmentally dependent and the way selection influences mortality rates may well differ between field and laboratory conditions.

In conclusion, the field cage experiments suggest that Wolbachia effects will depend on the nuclear background of its host D. melanogaster. Overall, the results emphasize the fact that laboratory and field experiments with Drosophila can lead to different conclusions even in this rather simplified version of the field environment. It is possible that even more complex interactions are occurring between D. melanogaster and Wolbachia in the field environment that were not detectable here.

\section{Acknowledgements}

This work was supported by the Australian Research Council via their Large Grant and Special Research Centre programmes. 


\section{References}

BOULETREAU-MERLE，J., FOUILLET, P. AND TERRIER, O. 1992. Clinal and seasonal variations in initial retention capacity of virgin Drosophila melanogaster females as a strategy for fitness. Evol. Ecol., 6, 223-242.

BOURTZIS, K., NIRGIANAKI, A., MARKAKIS, G. AND SAVAKIS, C. 1996. Wolbachia infection and cytoplasmic incompatibility in Drosophila species. Genetics, 144, 1063-1073.

FUKUI, H. H., XIU, L. AND CURTSINGER, J. W. 1993. Slowing of age-specific mortality rates in Drosophila melanogaster. Exp. Gerontol., 28, 585-599.

GIORDANO, R., O'NEILL, S. L. AND ROBERTSON, H. M. 1995. Wolbachia infections and the expression of cytoplasmic incompatibility in Drosophila sechellia and D. mauritiana. Genetics, 140, 1307-1317.

HOFFMANN, A. A. AND HARShMAN, L. G. 1999. Desiccation and starvation resistance in Drosophila: patterns of variation at the species, population and intrapopulation levels. Heredity, 83, 637-643.

hofFMANN, A. A. AND TURELli, M. 1997. Cytoplasmic incompatibility in insects. In: O'Neill, S. L., Hoffmann, A. A. and Werren, J. H. (eds) Influential Passengers: Microorganisms and Invertebrate Reproduction, pp. 3-80. Oxford University Press, Oxford.

hofFMANN, A. A., TURElli, M. AND haRShMAN, L. G. 1990. Factors affecting the distribution of cytoplasmic incompatibility in Drosophila simulans. Genetics, 126, 933-948.

HOFFMANN, A. A., ClANCY, D. J. AND MERTON, E. 1994. Cytoplasmic incompatibility in Australian populations of Drosophila melanogaster. Genetics, 136, 993-999.

HOFFMANN, A. A., HERCUS, M. AND DAGHER, H. 1998. Population dynamics of the Wolbachia infection causing cytoplasmic incompatibility in Drosophila melanogaster. Genetics, 148, 221-231.

IZQUIEREDO, J. I. 1991. How does Drosophila melanogaster overwinter? Entomologia exp. appl., 59, 51-58.

JEYAPRAKASH, A. AND HOY, M. A. 2000. Long PCR improves Wolbachia DNA amplification: wsp sequences found in $76 \%$ of sixty-three arthropod species. Insect Mol. Biol., 9, 393-405.
JOHANOWICZ, D. L. AND HOY, M. A. 1999. Wolbachia infection dynamics in experimental laboratory populations of Metaseiulus occidentalis. Entomologia exp. appl., 93, 259-268.

MERCOT, H. 1997. Wolbachia infection in Drosophila simulans - does the female host bear a physiological cost? Evolution, 51, 180-186.

O'NEILL, S. L., GIORDANO, R., COLBERT, A. M. E., KARR, T. L. ET AL. 1992. 16S rRNA phylogenetic analysis of the bacterial endosymbionts associated with cytoplasmic incompatibility in insects. Proc. Natl. Acad. Sci. U.S.A., 89, 2699-2702.

PARTridge, L., Prowse, N. AND Pignatelli, P. 1999. Another set of responses and correlated responses to selection on age at reproduction in Drosophila melanogaster. Proc. R. Soc. B, 266, 255-261.

PERROT-MINNOT, M. J., GUO, L. R. AND WERREN, J. H. 1996. Single and double infections with Wolbachia in the parasitic wasp Nasonia vitripennis - effects on compatibility. Genetics, 143, 961-972.

PROMISLOW, D.E. L., TATAR, M., KHAZAELI, A. A. AND CURTSINGER, J. W. 1996. Age-specific patterns of genetic variance in Drosophila melanogaster. I. Mortality. Genetics, 143, 839-848.

ROSE, M. R. AND CHARLESWORTh, B. 1981. Genetics of life history in Drosophila melanogaster. II. Exploratory selection experiments. Genetics, 97, 187-196.

SCHOENMAKER, A., VANDENBOSCH, F. AND STOUTHAMER, R. 1998. Symbiotic bacteria in parasitoid populations - coexistence of Wolbachia-infected and uninfected Trichogramma. Oikos, 81, 587-597.

TURELl, M. AND HOFFMANN, A. A. 1995. Cytoplasmic incompatibility in Drosophila simulans - dynamics and parameter estimates from natural populations. Genetics, 140, 1319-1338.

VOelKer, R. A., GIBSON, W., GRAVES, J. P., STERLING, J. F. ET AL. 1991. The Drosophila suppressor of sable gene encodes a polypeptide with regions similar to those of RNA-binding proteins. Mol. Cell. Biol., 11, 894-905.

WERren, J. H. 1997. Biology of Wolbachia. Ann. Rev. Entomol., 42, 587-609. 